FORMATION Formation emploi

Revue française de sciences sociales

120 | Octobre-Décembre 2012

Parcours de formation : la recomposition des ségrégations

\title{
Les métiers manuels, incarnation suprême du « bon travail »?
}

Jean-Paul Cadet

\section{(2) OpenEdition}

Journals

Édition électronique

URL : https://journals.openedition.org/formationemploi/3872

DOI : $10.4000 /$ formationemploi.3872

ISSN : 2107-0946

Éditeur

La Documentation française

Édition imprimée

Date de publication : 31 octobre 2012

Pagination : 147-153

ISSN : 0759-6340

Référence électronique

Jean-Paul Cadet, «Les métiers manuels, incarnation suprême du « bon travail »? », Formation emploi [En ligne], 120 | Octobre-Décembre 2012, mis en ligne le 08 janvier 2015, consulté le 21 septembre 2021. URL : http://journals.openedition.org/formationemploi/3872 ; DOI : https://doi.org/10.4000/ formationemploi.3872 


\title{
Note de lecture
}

\section{Les métiers manuels, incarnation suprême du «bon travail»?}

\author{
Présentation de l'ouvrage \\ Eloge du carburateur. Essai sur le sens et \\ la valeur du travail
}

par Jean-Paul Cadet Chargé d'études au Céreq

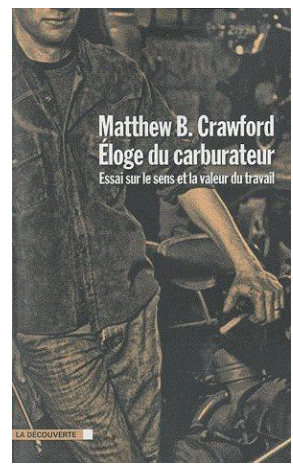

Qu'est-ce qu'un «bon travail » ou un emploi de qualité ? Si cette question d'ordre philosophique ne peut appeler une réponse simple et uniforme, elle n'est pourtant plus à éluder. Plus que jamais, il est aujourd'hui admis que le travail peut être à l'origine d'importants maux (maladies professionnelles, « risques psycho-sociaux »...), susceptibles eux-mêmes d'engendrer tout un ensemble de coûts plus ou moins cachés pour les organisations (non-qualité, absentéisme, démotivation...). De même, l'expérience du travail contemporain est loin de rimer systématiquement avec sécurité, stabilité, reconnaissance et progression. Cette expérience est souvent au contraire synonyme de précarisation, de déprofessionnalisation, de mise en péril des devenirs personnels.

De façon générale, on peut dire que l'emploi de qualité repose d'abord et avant tout sur la notion de sens. Idéalement, un travail est doté de sens quand il offre tout à la fois un sentiment d'utilité, des occasions d'apprentissage, une appartenance sociale, une possibilité de se réaliser, un revenu décent, un statut, des perspectives d'évolution ou à tout le moins une sécurité d'emploi. Partant, le problème est d'identifier les situations de travail qui se rapprochent le plus de ce modèle idéal, et inversement celles qui s’en éloignent aussi le plus. L'enjeu réside dans la promotion des premières et la transformation des secondes pour lutter contre les maux précités et réduire les coûts associés.

Pour Matthew B. Crawford, auteur nord-américain, il n'y a pas de doute. Les métiers manuels " artisanaux " font partie des plus signifiants. Par ces métiers, il entend tous ceux qui ont trait à une activité de fabrication ou de réparation d'objets matériels, et qui portent encore en eux l'esprit artisanal. Plombier, maçon, électricien, mécanicien... : ces métiers sont censés déborder de vertus cognitives, sociales et psychologiques pour ceux qui en font l'expérience. Ils sont d'ailleurs loin d'avoir disparu de nos jours. Beaucoup d'entre 
eux se caractérisent au contraire par de constantes et importantes pénuries. En cherchant à réhabiliter ce genre de travail manuel, l'auteur nous propose alors une réflexion stimulante sur ce qui est source de sens et de valeur dans les activités professionnelles au sein de nos sociétés modernes. Ce faisant, il tente de repérer les aspects du travail et de la formation qui vont en ce sens et, à l'inverse, ceux qui s'inscrivent dans une direction opposée.

Matthew B. Crawford sait de quoi il parle. Philosophe, il est aussi réparateur de motos. Il a même renoncé à une carrière brillante d'universitaire et de directeur d'un think tank en reprenant un atelier de réparation de motos. Pour développer ses thèses, il s'appuie sur cette surprenante bifurcation professionnelle, et plus largement sur toute son expérience vécue en tant que travailleur manuel - tout en faisant ses études, il avait déjà été notamment mécanicien automobile. Il restitue avec talent et non sans humour cette expérience, en la mettant en perspective avec des réflexions philosophiques et sociologiques plus globales. Iconoclaste, la méthode s'avère au final convaincante et savoureuse.

\section{1| Le travail manuel, comme vous ne l'avez sans doute jamais imaginé}

"Mettre les mains dans le cambouis n'a rien de dégradant. Bien au contraire ". Les métiers manuels artisanaux représentent en effet des activités qui ont le mérite d'être clairement orientées vers une fin précise (fabriquer ou réparer tel objet) et réalisées de manière intégrale (le professionnel prend en main tout un processus). De plus, ce sont des activités extrêmement riches sur le plan cognitif. Elles ne peuvent jamais être réduites à l'application de règles prédéfinies et définitives. Elles requièrent toujours de l'intelligence en situation et une expertise spécifique. Par exemple, une vertu majeure du travail manuel est de solliciter énormément l'attention du travailleur - surtout dans les activités de réparation où il s'agit de décrypter des pannes ou des grippages avant d'agir. Les métiers manuels ont également pour qualité de procurer un fort sentiment de responsabilité. Selon l'auteur, le travailleur manuel exerce une activité aisément évaluable : "Sur un chantier, vous disposez de critères objectifs pour évaluer votre propre contribution indépendamment des autres, et ce sont ces mêmes critères qui serviront à vos camarades de travail pour vous juger. Soit vous êtes capable de courber un câble rigide, soit vous ne l'êtes pas, il n'y a pas d'échappatoire. " (p. 180) Le travailleur manuel se distingue aussi par une forte indépendance d'esprit.

Cette indépendance d'esprit n'a pourtant rien à voir avec un repli sur soi. En effet, le travail manuel s'inscrit toujours dans un cadre communautaire. D'une part, il est inséparable d'un groupe de pairs donné ou d'une « communauté de pratiques » comme diraient certains ${ }^{1}$. Ce sont notamment les pairs qui sont les plus compétents pour reconnaître la

1. Cf. Etienne Wenger, Communities of Practice : Learning, meaning and identity, Cambridge University Press, 1998. 
qualité d'un travail manuel. D'autre part, ce type d'activité est indissociable d'une communauté de clients. Ceux qui exercent pareille activité ont des relations directes avec des clients, et ces relations sont de nouvelles occasions pour évaluer leur prestation. La richesse du travail manuel artisanal s'apprécie donc aussi à l'aune de sa dimension sociale.

Le bénéfice psychique qu'il fournit n'est pas non plus négligeable. C'est un travail qui se révèle le plus souvent captivant. Il offre la possibilité d'occuper une place utile et reconnue dans la société. Il procure surtout un vif sentiment de plaisir au travail, en permettant au travailleur de s'engager à fond dans son activité, avec une impression gratifiante de compétence, "comme Nadia Comaneci sur sa barre d'exercice " (p. 224). Electricien à un moment donné, l'auteur raconte par exemple : "Le moment où, à la fin de mon travail, j’appuyais enfin sur l'interrupteur ("Et la lumière fut ") était pour moi une source perpétuelle de satisfaction. J'avais là une preuve tangible de l'efficacité de mon intervention et de ma compétence. Les conséquences de mon travail étaient visibles aux yeux de tous, et donc personne ne pouvait douter de ladite compétence. Sa valeur sociale était indéniable.» (p. 20)

Les métiers manuels sont par ailleurs associés à un mode de formation fondé sur l'acquisition progressive et patiente d'une expérience pratique. L'auteur soutient l'idée, à la fois banale et irréfutable, que le savoir-faire ne peut naître que d'une confrontation répétée avec le réel. Il s'en est directement aperçu quant il s'employait à acquérir son propre savoirfaire : "Je reconnaissais implicitement que ma vigilance professionnelle laissait beaucoup à désirer : j'étais encore loin de pouvoir passer pour une personne lucide dotée d'un champ de vision ample et capable de percevoir la situation dans toutes ses dimensions. Ce sont là des qualités qui ne sont susceptibles d'être acquises que progressivement, au gré des circonstances. " (p. 144) C'est une telle acquisition via l'expérience qui permet aux apprentis travailleurs manuels de s'approprier les principales exigences de leur métier, comme le fait, par exemple, d'être capable de discipliner son attention, ou d'intégrer à sa pratique la possibilité de l'échec ou de l'erreur (surtout lorsqu'il s'agit de réparer un objet).

De nature singulière, semblable expérience nécessite cependant la présence d'un autrui. Pour les apprentis, ce sera principalement un aîné de la profession ou un « maître ». C'est lui qui leur transmettra les ficelles du métier, les bonnes raisons d'agir de telle ou telle manière dans telle ou telle situation, les conseils utiles à suivre pour développer leur pratique. Pour tout travailleur confirmé, les pairs, et notamment "les plus excellents", seront la ressource indispensable pour améliorer le savoir-faire. Les discussions avec les pairs se révèleront particulièrement fécondes quand le travailleur butera sur une difficulté. Elles conditionneront donc la possibilité pour chacun de progresser dans son art, sinon de s'orienter vers l'excellence - une condition de la «vie bonne» pour Matthew B. Crawford. 


\section{Un travail « intellectuel » qui apparaît bien pauvre en comparaison}

Face aux métiers manuels artisanaux, les métiers exercés au sein des grandes organisations font pâle figure, nous dit l'auteur. Ils souffrent pour l'essentiel de la dissociation entre le " penser » et le « faire » ou entre la conception et l'exécution, instaurée au siècle dernier par l'industrie taylorienne et fordienne. Cette dissociation a conduit à démembrer les activités de travail en au moins deux grandes parties : une partie " manuelle », dégradée, dévalorisée et confiée à des ouvriers ou " cols bleus » expropriés de leur savoir-faire traditionnel ; une partie " intellectuelle », valorisée et prise en charge par des " cols blancs » (managers, ingénieurs...), porteurs du seul savoir reconnu, supposé d'ordre technico-scientifique.

L'intérêt du propos de Crawford est alors de montrer que les cols blancs sont en fait euxmêmes victimes d'un processus de dégradation de leur travail. En regard du travail manuel artisanal, leur activité - dite de "bureau » - apparaît beaucoup moins signifiante, dès lors qu'elle est prise isolément et sortie de son contexte organisationnel. Elle offre aussi moins de possibilités de se montrer créatif et ne permet pas de construire des liens aussi forts de coopération et de solidarité entre pairs. Pour l'auteur, elle manque surtout de critères objectifs pour évaluer sa qualité et les personnels qui l'exercent. Les tâches liées à ce travail sont à vrai dire floues et peu à même d'être soumises à une sanction indiscutable, comme celle du fil à plomb pour le maçon. De là le sentiment d'irresponsabilité, sinon d'infaillibilité qui caractérise l'attitude de nombreux travailleurs intellectuels (surtout les managers). De là également le primat qu'ils accordent à leur image et à la qualité des relations qu'ils nouent avec leurs supérieurs hiérarchiques par rapport à leur compétence effective. De là enfin l'ambiguïté et l'arbitraire qui enveloppent la gestion de leur carrière au sein des grandes organisations.

Les organisations du travail issues $\mathrm{du}$ "nouveau capitalisme », censées rompre avec les rigidités du taylorisme et du fordisme, sont loin de remettre en cause cette dégradation du travail de bureau. Au contraire, elles mettent davantage en avant les qualités personnelles des travailleurs par rapport à leur savoir-faire : leur "affabilité ", leur " complaisance "... Elles demandent à leurs personnels de s'identifier plus à leur culture qu'à des cultures de métiers spécifiques. Elles font régner à vrai dire la polyvalence et la flexibilité, qui sont aux antipodes de l'identité professionnelle du travailleur manuel fondée sur l'acquisition patiente d'une expérience dans un domaine précis.

Quant aux pratiques éducatives ayant vocation à préparer au travail " intellectuel ", elles apparaissent problématiques aux yeux de l'auteur. Elles renvoient aux études universitaires et aux diplômes correspondants. Elles sont d'autant plus privilégiées aujourd'hui que la " nouvelle économie " est censée avoir besoin de " travailleurs de la connaissance " aptes à apprendre constamment des choses nouvelles et à faire preuve de flexibilité. Mais elles contribuent à accorder un poids excessif aux diplômes universitaires. Elles provoquent, selon le sociologue Collins, une dynamique d'inflation des diplômes "apparemment sans 
fin, jusqu'au jour où il faudra un doctorat pour être concierge et où les baby-sitters ne pourront pas travailler sans un diplôme avancé de puériculture" (cité p. 165)². En outre, ces pratiques ne sont pas sans effets pervers. Plus que les contenus dispensés, les notes, les unités de valeur et les diplômes deviennent les principaux objectifs des étudiants. Et, en termes de connaissances à acquérir, le "savoir comment " passe à la trappe au profit du seul "savoir que».

Dans ce contexte, l'auteur doute de la capacité des études supérieures à former des jeunes vraiment indépendants, créatifs, épanouis, humbles, honnêtes, en prise avec le réel... à l'image de ce que permet le travail manuel. En particulier, l'expérience de l'échec, pourtant si formatrice, ne semble pas leur être inculquée. L'auteur va jusqu’à juger risquée la fréquentation de l'université du point de vue de l'employabilité. Il s'appuie alors sur cette intuition provocatrice de Schumpeter datant de 1942 : «L'homme qui a fréquenté un lycée ou une université devient psychiquement inemployable dans des occupations manuelles sans être devenu pour autant employable, par exemple, dans les professions libérales." (cité p. 149), (comme celles d'avocat ou de médecin)

\section{Une approche à relativiser}

L'approche de Crawford est certainement à relativiser. Tout d'abord, il n'est pas sûr que les métiers manuels aient le monopole du bon travail. Bien d'autres activités, dotées a priori de sens, pourraient figurer parmi la liste des emplois de qualité. Citons entre autres exemples les emplois qualifiés d'aide à la personne. Après tout, l'auteur se limite à opposer le travail manuel artisanal au travail intellectuel exercé au sein des grandes organisations. Il laisse plus ou moins de côté toutes les autres occupations ou professions. Il n'est d'ailleurs pas dupe à ce sujet : "Il existe certainement d'autres types de tâches dont je ne suis pas familier et où ces biens intrinsèques peuvent être réalisés ; je laisse à d'autres le soin de les explorer." (p. 230)

Plus généralement, ne peut-on pas éprouver dans d'autres univers ou activités le même type de satisfaction et d'épanouissement que celui que l'on ressent en exerçant un travail manuel ? En fait, les sentiments d'utilité, de compétence, de responsabilité peuvent caractériser, à des degrés divers, de très nombreuses situations de travail. La qualité d'un même emploi peut sensiblement varier selon les conditions technico-économiques et organisationnelles, plus ou moins favorables, dans lesquelles il émerge et se déploie. D'ailleurs, les situations de travail manuel ne sont pas elles-mêmes à l'abri de conditions défavorables. Certaines ne voient-elles pas se standardiser une partie de leurs tâches (développement

2. Ce point dénoncé par Crawford n’est pas sans rappeler certaines analyses développées en France. Voir par exemple Marie Duru-Bellat, Linflation scolaire, coll. « La République des idées », éd. du Seuil, 2006. 
du " prêt-à-poser » dans les activités de maintenance, rationalisation des technologies de diagnostic...) ? De plus, tout n'est pas idéal dans leurs conditions d'exercice, comme le montrent, par exemple, les difficultés rencontrées par de nombreux jeunes apprentis, amenés à rompre leur contrat d'apprentissage ${ }^{3}$. Cette prise en compte des contextes rencontrés par les travailleurs invite au total à adopter une approche moins essentialiste que celle de l'auteur, et plus sensible aux conditions concrètes qui leur sont offertes, ou non, pour se réaliser et développer leur pouvoir d'agir .

Ensuite, il y a lieu d'évoquer un effet d'ordre sociétal. Au vu des éléments avancés par l'auteur, il est très probable que le travail manuel soit encore plus malmené aux États-Unis qu'en France, notamment depuis les années 80. En France, l'orientation vers ce type de travail s'opère encore largement par l'échec, alors que l'on assiste en même temps à une massification de l'enseignement supérieur ; toutefois, depuis plusieurs années, on tente de revaloriser le travail manuel ${ }^{5}$, via en particulier la mise en avant fréquente des pénuries qui le concernent sur le marché du travail et les efforts salutaires menés par le système de formation professionnelle (discours et pratiques en faveur de l'alternance, création du bac pro dans les années $80 \ldots$...

On peut regretter par ailleurs le penchant objectiviste et élitiste de l'auteur. Pour lui, les métiers manuels sont vertueux, entre autres parce qu'ils reposent sur des critères d'évaluation a priori clairs et objectifs, ainsi que sur une communauté professionnelle assise sur une hiérarchie dominée par les aînés et les plus expérimentés. Or, si contrairement à nombre d'activités relationnelles et de bureau, le travail manuel se prête à des appréciations plus aisées de performance (par exemple, réussir ou pas à diagnostiquer une panne), les critères qui déterminent le " travail bien fait ", le " geste professionnel " correctement réalisé ou la bonne démarche à suivre demeurent constamment incertains, sujets à discussion et au changement ${ }^{6}$. Du reste, l'innovation en question, le renouvellement des conceptions n'ont pas à émaner seulement des professionnels qui trônent, à un moment donné, au sommet de la hiérarchie de la communauté. Tous les praticiens devraient avoir leur mot à dire, même les plus jeunes ou les plus « originaux ». De fait, pour qu'il puisse être vecteur au mieux de sens, le travail devrait être compris et organisé comme une agora, où l'on peut discuter et réviser régulièrement les critères de sa qualité et de sa justesse ${ }^{7}$, à

3. Voir à ce propos Benoît Cart, Marie-Hélène Toutin et Valérie Henguelle, «Contrat d'apprentissage, les raisons de la rupture ", Bref Céreq, n² 272, 2010.

4. On rejoint là, dans une certaine mesure, l'approche dite par les " capabilités "; cf. Amartya Sen, Ethique et économie, PUF, 2008.

5. Remémorons-nous, par exemple, la politique impulsée au milieu des années 70 par Lionel Stoléru, alors secrétaire d'État à la Condition des travailleurs manuels.

6. Citons le cas typique des métiers du bâtiment, où pareilles discussion et dynamique vont jusqu’à conditionner la prévention et la limitation des risques professionnels ; voir à ce sujet Pierre Trinquet, Prévenir les dégâts du travail : l'ergoprévention, PUF, 2009.

7. Cf. Yves Clot, Le travail à cœur : pour en finir avec les risques psycho-sociaux, La Découverte, 2010. 
partir notamment de cadres de références et de " principes de justification " spécifiques ${ }^{8}$ plutôt que d'être considéré et traité comme une activité se référant strictement à un ordre professionnel établi et immuable.

Enfin, force est de reconnaître que l'analyse des études universitaires brossée par Crawford frise la caricature. Si cette analyse vise, par effet de contraste, à promouvoir le mode d'apprentissage et de socialisation professionnelle en vigueur au sein des métiers manuels, elle exagère largement la critique des études supérieures et la portée de l'inflation scolaire. Globalement, ces études n'ont-elles pas sans cesse montré, ces dernières années, qu'elles restaient, pour la majorité des jeunes, la meilleure arme anti-chômage ? De plus, ne se sont-elles pas en partie " professionnalisées ", c'est-à-dire ouvertes davantage sur des champs de métiers en particulier et sur le monde du travail en général ?

Louvrage de Matthew B. Crawford n'en demeure pas moins une œuvre originale incontournable pour toux ceux et celles qui s'intéressent au travail et à ses implications sur les systèmes de formation et d'éducation au sein des sociétés occidentales. Il souligne à juste titre les vertus, l'éthique et même l'esthétisme du travail manuel - à l'image, comme l'évoque l'auteur, du "plombier accroupi sous l'évier, la raie des fesses à l'air». Les métiers manuels sont dès lors présentés comme parties intégrantes du « bon travail », celui-ci pouvant donc être là où on ne l'attend pas forcément. Ils bénéficient en définitive d'un plaidoyer, doublement utile. D’une part, ce plaidoyer joue comme une réhabilitation, alors que le travail manuel reste encore trop souvent décrié et fui injustement par de nombreux élèves et leurs familles au moment de l'orientation scolaire. D'autre part, un tel plaidoyer, favorable à un modèle professionnel - celui du " métier " - que d'aucuns ont cru à un moment donné dépassé, sonne juste à l'heure où les autres grands modèles concurrents celui du " poste de travail » au sein des organisations tayloriennes et fordiennes, et celui de la " compétence ", dans le cadre des organisations issues du nouveau capitalisme - ont montré leurs limites ${ }^{9}$. Sans être exhaustif, l'ouvrage invite alors à engager et à poursuivre sans relâche une recherche sur les constituants et les conditions du bon travail. Soit un projet de recherche-action on ne peut plus urgent aujourd'hui, à l'heure où s'amplifient la maltraitance du travail et par conséquent celle des travailleurs.

\section{Référence de l'ouvrage}

Crawford M.B. (2010), Éloge du carburateur. Essai sur le sens et la valeur du travail ? Editions La Découverte, Paris, p. 249.

8. Cf. Boltanski L., Thévenot L. (dir.), Justesse et justice dans le travail, Cahiers du Centre d'études de l'emploi, $\mathrm{n}^{\circ}$ 33, Paris, PUF, 1989.

9. Pour une présentation et une analyse de ces trois modèles, cf. Philippe Zarifian (2004), Le modèle de la compétence. Trajectoire historique, enjeux actuels et perspectives, $2^{\text {ème }}$ édition actualisée, Editions Liaisons. 